Trauma Berufskrankh 2018 20:229

https://doi.org/10.1007/s10039-018-0408-5

(c) Springer Medizin Verlag GmbH, ein Teil von Springer Nature 2018

CrossMark

Fabian M. Stuby

BG-Unfallklinik Murnau, Murnau am Staffelsee, Deutschland

\title{
Verletzungen der proximalen Tibia
}

Die vorliegende Ausgabe der Zeitschrift Trauma \& Berufskrankheit beschäftigt sich schwerpunktmäßig mit der Tibiakopffraktur und soll dem Leser in ausgewählten Artikeln die Möglichkeit geben, sich umfassend über das Thema $\mathrm{zu}$ informieren. In Anlehnung an die zeitliche Reihenfolge des Geschehens haben die Autoren jeweils umfassend ihre Themen für den Leser bearbeitet und aufbereitet. Von den Entstehungsmechanismen und biomechanischen Grundlagen über die Klassifikation und Therapieentscheidung, die bei operativer Therapie notwendigen Grundlagen der verschiedenen Zugangswege sowie der Prinzipien der Osteosynthese an der proximalen Tibia bis hin zu den Ergebnissen nach Behandlung dieser Verletzung bietet das vorliegende Heft eine übersichtliche Zusammenfassung dieses Themas an. Der Leser kann sich somit entweder ein gutes Grundlagenwissen durch die Bearbeitung aller Artikel verschaffen oder kann in einzelnen Bereichen eine Vertiefung des mitunter bereits vorhandenen Erfahrungsschatzes erlangen.

Sowohl die in den letzten Jahren zunehmend angewendeten CT-basierten Klassifikationen als auch die aufgrund der immer häufiger diagnostizierten dorsalen Beteiligung des Tibiaplateaus notwendigen posterioren Zugangswege werden beschrieben, sodass sich der Leser nach Lektüre der Zeitschrift auf dem aktuellem Wissensstand in Bezug auf die Behandlung der Tibiakopffrakturen befindet.

Des Weiteren können wir Ihnen im vorliegenden Heft die zwischenzeitlich als Standardverfahren mit deutlich geringerer Komplikationsrate etablierte minimalinvasive Versorgung von Kalkaneus- frakturen präsentieren. Diese kann sowohl unter Verwendung bereits vorhandener und etablierter Implantate als auch unter Verwendung von eigens für diesen Zugangsweg neu entwickelten Platten durchgeführt werden.

Wie auf allen Gebieten, so befindet sich die moderne Unfallchirurgie auch im Rahmen der hier abgehandelten 2 verschiedenen Verletzungsentitäten in einem ständigen Entwicklungsprozess, sodass wir gespannt sein können, wie sich die Versorgung dieser Verletzungen in den nächsten Jahren verändern wird.

Murnau, im November 2018
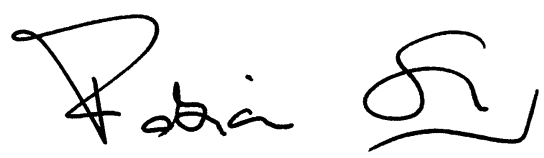

PD Dr. med. Fabian M. Stuby

\section{Korrespondenzadresse}

\section{PD Dr. med. Fabian M. Stuby}

BG-Unfallklinik Murnau

Prof.-Küntscher-Str. 8, 82418 Murnau am

Staffelsee, Deutschland

Fabian.Stuby@bgu-murnau.de

Interessenkonflikt. F.M. Stuby gibt an, dass kein Interessenkonflikt besteht. 